\title{
Fixed Set Point Weighting 2DOF PID Controller for Control Processes
}

\author{
Bahaaeldin Gamal Abdelaty ${ }^{*}$, Ahmed Hassan Ahmed, Ahmed Nasr Ouda \\ Electrical Engineering Department, Technical Research Center, Cairo, Egypt \\ Email address: \\ bahaa.blackeagel@yahoo.com (B.G. Abdelaty),ahmed_hassan_aircraft@yahoo.com (A.H. Ahmed), ahnasroda@yahoo.com(A. N. Ouda) \\ ${ }^{*}$ Corresponding author
}

To cite this article:

Bahaaeldin Gamal Abdelaty, Ahmed Hassan Ahmed, Ahmed Nasr Ouda. Fixed Set Point Weighting 2DOF PID Controller for Control Processes. Engineering Mathematics. Vol. 2, No. 1, 2018, pp. 21-27. doi: 10.11648/j.engmath.20180201.13

Received: June 4, 2018; Accepted: June 21, 2018; Published: July 12, 2018

\begin{abstract}
Nowadays, many of control systems need a flexible controller in order to achieve the operation requirement precisely and rapidly to overcome nonlinearity, uncertainty, digital implementation challenges, and achieving at the same time both of control system objectives of load disturbance rejection and set point following. This paper is aimed to design and analysis one of the most common industrial controllers, set point weighting 2DOF PID controller, to get this target with simplicity and effectiveness. The paper included the main problem description, structure of the proposed controller, effectiveness of controller parameters variations, the methodology of effective tuning method, and an example of the proposed controller on control system platform. The results reveal the accurate and effective operational performance of the proposed controller to obtain the control system objectives.
\end{abstract}

Keywords: Set Point Weighting, 2DOF PID Controller, Control Systems, Control Objectives

\section{Introduction}

In the field of control engineering, a primary objective of a control theory is to adjust the output of a dynamic process behave in a certain manner [1]. Therefore, various control strategies are implemented, devised, and experienced in the dynamic process control applications. However, the majority of engineering problems in different fundamental engineering domain today are come more complex, broader, and deeper. As well as, solving and managing complexity, uncertainty, and communication through the design of the control process is a very critical issue, especially system nonlinearities and uncertainties in order to have exceptional levels of accuracy and speed including the ability to perform complicated and precise movements of high quality $[2,3]$.

Each controller has its advantages and disadvantages. Therefore, the designer must select the appropriate controller to meet all the design specifications, including simplicity of structure, cost, reliability, development techniques, and digital hardware implementation $[4,5]$. As well as, there are many factors might influence the selection process including integration, space, processor power, robustness, safety criteria of the application. Therefore, choosing a specific controller for a specific application is often based on the designer's experience, the accuracy of the derived mathematical model, the accuracy and limitations of applied design technique. Besides that variety of control techniques that may be more or less appropriate to a specific type of applications, such as PID control, robust control (H- infinity loop shaping, sliding mode control), adaptive control, optimal control, intelligent control, etc. offer a widespread area of control selection [6].

A typical problem with the design a feedback controller is to achieve at the same time a high performance in the set point following and in the load disturbance rejection [7]. In addition, at the same time, from digital implementation viewpoint and its usefulness for developing the control algorithm performance, mostly of researchers all over the world prefer using Proportional, Integral, and Derivative architecture in parallel or series form so far. There are several reasons for preferring PID controllers, including simplicity of structure, satisfied performance for most cases, and obvious tuning technique $[8,9]$.

The purpose of this article is to summarize main points on design and analysis of set point weighting (Filter type) 2DOF PID controller, so that any interested one in this topic can easily understand, and exploit the results. 


\section{Main Problem Description}

In the past, in the most industrial application, the desired value (set point) normally remains constant and a good disturbance rejection is required, which can be described as regulator control. However, due to great variation in the process conditions at the last decades, the control variable set point may eventually need to be altered and then a suitable transient response is required. Under this circumstances, satisfying required operating conditions is difficult by using one-degree-of-freedom (abbreviated as 1DOF) PI/PID control, but using two-degree-of-freedom (abbreviated as 2DOF) gives a chance to tune a controller performance in order to achieve a required control performance $[8,10]$.

From both mathematical and computation point of view, easy implementation and adequate performances are the main features of both 1DOF and 2DOF PID controller [11]. However, tracking with classical PID controllers over the operating range of highly nonlinear uncertain systems is difficult to achieve with excellent performances. In other words, for such systems, classical PID controllers lack their credibility, reliability, and robustness. The 2DOF controller is the best choice to obtain the advanced control requirements [12-15].

\section{Insufficiency of PID Controller}

Under some assumptions like the sensors has a good accuracy and speed in the steady state operation condition and the main disturbance enters at the manipulating point as the input disturbance on the system, the structure of classical PID control system can be presented as shown in Figure 1.

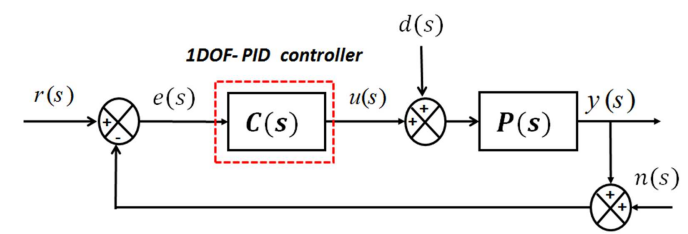

Figure 1. Classical PID controller under specific assumptions.

The closed-loop transfer function of the overall system can be represented from the set point $r(s)$ to the controlled variable $y(s)$ and from the disturbance point $d(s)$ to $y(s)$ are, respectively, given by

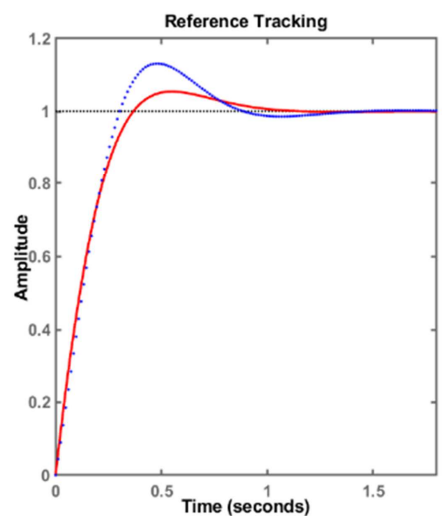

$$
\begin{gathered}
G_{y r}(s)=\frac{y(s)}{r(s)}=\frac{P(s) C(s)}{1+P(s) C(s)} \\
G_{y d}(s)=\frac{P(s)}{1+P(s) C(s)}
\end{gathered}
$$

Where $P(s)$ a mathematical model of the plant, $C(s)$ is a PID controller. These transfer function included only one variable element is $C(s)$, so both of them cannot be changed independently. Therefore, if the load disturbance rejection response is optimized, the set point following has deviated, and vice versa.

By given a numerical example, suppose that the plant is a mathematical model of DC motor, the goal of the overall design is to track a reference change with minimal overshoot and to reject input disturbances. The controller must satisfy the control system requirements, including overshoot less than $6 \%$, settling time less than 1.5 seconds, rising time less than 0.7 second, and The output $(y)$ of a unit step input disturbance is reduced by $80 \%$ within 0.3 seconds and by $95 \%$ within 0.65 second [16-18]. The transfer function of the mathematical model of the DC motor describes as the following:

$$
P(s)=\frac{10}{s^{2}+14 * s+41}
$$

First, for having a required disturbance rejection, the classical PID parameters are obtained by Ziegler-Nichols tuning formula to be as the following:

$$
K_{p}=17.46, K_{i}=78.32, K_{d}=0.86
$$

As a resultant of these parameters, the disturbance rejection performance is achieve the control system target but on the other hand the set point following suffered from high overshoot over than $13 \%$.

By changing the controller parameters to compensate the set point response deviation, the controller parameters will be as the following:

$$
K_{p}=8.62, K_{i}=36.53, K_{d}=0.51
$$

From the results, the set point following is accepted but the disturbance rejection response is out of range greatly. The graphical representation for both controllers is shown in Figure 2.

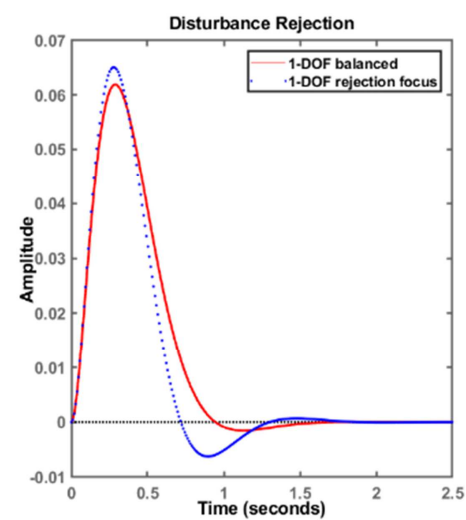

Figure 2. Time and disturbance rejection comparison between different 1DOF PID control objective. 
That entire argument makes the tuning methodology of the classical PID parameters limited by each control objective is more important and the rate of change of the set point variable $[7,19,20]$. For the previous reasons, the optimization between set point following and disturbance rejection cannot be achieved at the same time, so the researchers used for optimal PID control performance a tuning switch between one controller for set point following and the other for disturbance rejection.

To overcome these demerits, most of the researchers around the world started to put 2DOF PID controller as their first solutions. Using 2DOF PID controller offers a great opportunity to obtain an optimal response for both set point following and disturbance rejection practically at the same time, in addition, all of that in the linear framework as shown in the next section $[21,22]$.

\section{Set Point Weighting 2DOF PID Controller}

In the beginning, the meaning of 2DOF in control systems is representing the number of a closed-loop transfer function that can be obtained independently. Figure 3 shows 2DOF PID control structure that structure based on a combined feedforward/feedback controller [17, 23].

Generally, the 2DOF PID control structure has two inputs $r(s), y(s)$ to the single output $u(s)$ and it has different forms, all of them has the same performance, including feedforward type, feedback type, set point filter type, filter and proceeded derivative type, and components separate type. These controllers include set point weighting on the proportional and derivative terms. The error in an integral part must be actual control error to avoid steady-state control errors, therefore, no set point weight on the integral terms.

Set point filter type is one of the most common 2DOF PID forms, which used to cope the demerits of classical PID controller due to its simplicity and clear tuning methodology $[18,24,25]$. In the filter type configuration, the 2DOF PID controller is decomposed into a conventional SISO PID controller (feedback controller) $C(s)$ and a feed-forward filter on the reference signal $F(s)$ as shown in Figure 4.

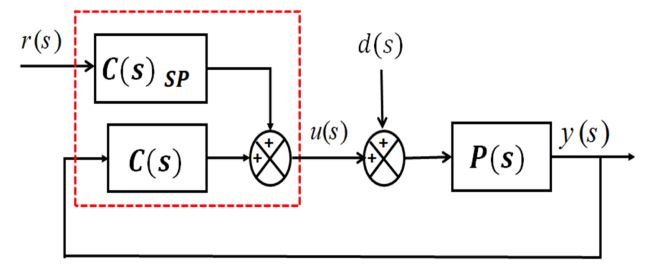

Figure 3. General 2DOF PID controller structure.

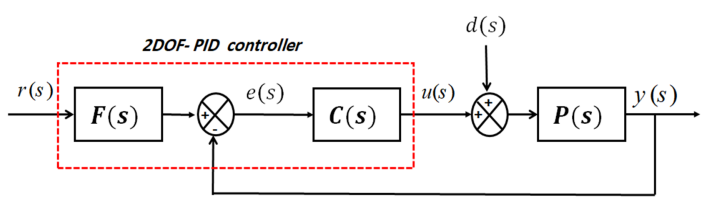

Figure 4. Set point weighting 2DOF PID structure.
The relationship between the 2DOF controller's output $(u)$ and its two inputs $(r$ and $y$ ) can be represented in the following equations:

$$
\begin{gathered}
u(s)=K_{p}\left(b r(s)-y(s)+\frac{K_{i}}{s}(r(s)-y(s))+\right. \\
\frac{K_{d} s}{T_{f} s+1}(\mathrm{c} r(s)-y(s)) \\
C(s)_{s p}=b K_{p}+\frac{K_{i}}{s}+\frac{c K_{d} s}{T_{f} s+1} \\
C(s)=-\left[K_{p}+\frac{K_{i}}{s}+\frac{K_{d} s}{T_{f} s+1}\right] \\
F(s)=\frac{K_{i}+\left(b K_{p}+T_{f} K_{i}\right) S+\left(b K_{p} T_{f}+c K_{d}\right) s^{2}}{K_{i}+\left(K_{p}+T_{f} K_{i}\right) S+\left(K_{p} T_{f}+K_{d}\right) s^{2}}=-\left[\frac{C(s)_{s p}}{C(s)}\right]
\end{gathered}
$$

Where $K_{p}, K_{i}, K_{d}$ PID classical control gains, $T_{f}$ derivative filter time, $b$ set point weight on the proportional term, and $c$ set point weight on the derivative term. Note that, both of the transfer function at Eq. $(7,8)$ from the input to the output is a PID controller with different weights. Consequently, it appears that the load disturbance rejection decoupled from the set point following one and obviously does not depend on the weights $b$ and $c[26,27]$.

Set point weighting parameter does not affect the response to the load disturbance rejection performance and measurement noise. This is applied to reduce the overshoot, steady-state error, in the output following step changes in the reference point [28-30]. Thus, the PID parameters can be select to achieve a high load disturbance rejection and then set point following performance can be recover by suitably selecting the value of the parameters $b$ and $c[8,14,28]$.

\section{The Methodology of Parameters Tuning}

The tuning parameters of the proposed controller are tuned in the following steps[24]:

a) Classical controller parameters $\left(K_{p}, K_{i}, K_{d}\right)$ are tuned for accepted load disturbance rejection performance (according to control system objectives).

b) Weights $(b, c)$ is adjusted in order to set zeros of the closed-loop transfer function and thus to improve the tracking behavior of the control system.

c) Usually, the value of the weights is between 0 and 1 .

d) In any case, in order to avoid time-consuming experiments, tuning rules have been devised to determine explicitly a suitable values of $b$ and $c$ starting from a model of the process.

e) In some PID controller implementation, in order to avoid derivative and proportional kicks, weights of derivative and proportional parts are set equal 0 , obtained a form of I-PD controller.

f) In some cases, 2DOF PID has obtained a form of ID-P controller when $b=0$ and $c=1$ and a form of PI-D controller when $b=1$ and $c=0$. 


\section{Simulation Results}

For all of the simulation presented here, the general consideration are taken into account. The comparison between effects of two different control objective classical 1DOF PID control parameters are presented in section (3). The results show that limitation of classical control to meet both of control system requirements due to higher integrated gain for high disturbance rejection that causes higher overshoot in the system performance over than required. In this section, simulation results for the proposed controller is introduced, including set point

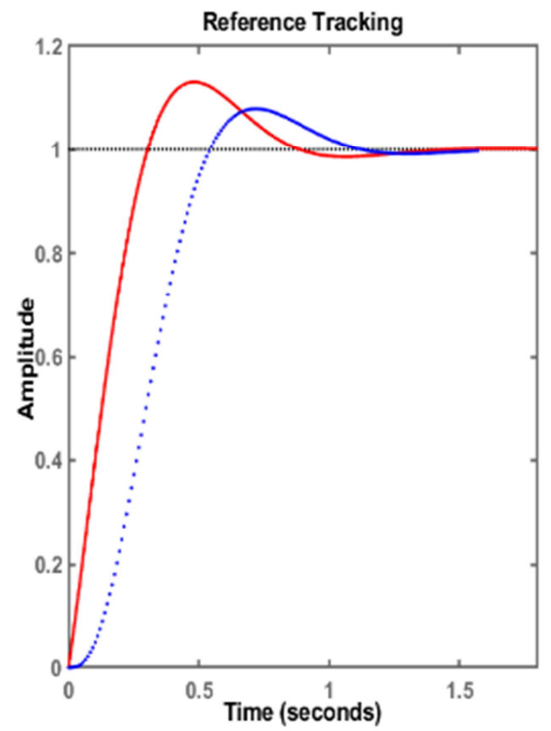

tracking reference variation, over all closed loop system response, variation of phase margin and its effectiveness, as well as, the setpoint weight values variation effect.

First, the comparison between the step response and disturbance rejection response between $1 \mathrm{DOF}$ and $2 \mathrm{DOF}$ PID controller with the same control objective, which is rejection focus, appears that capability of 2DOF PID controller to overcome system oscillation due to high integrating gain without any variation on the rejection performance. The graphical representation for both is shown in Figure 5.

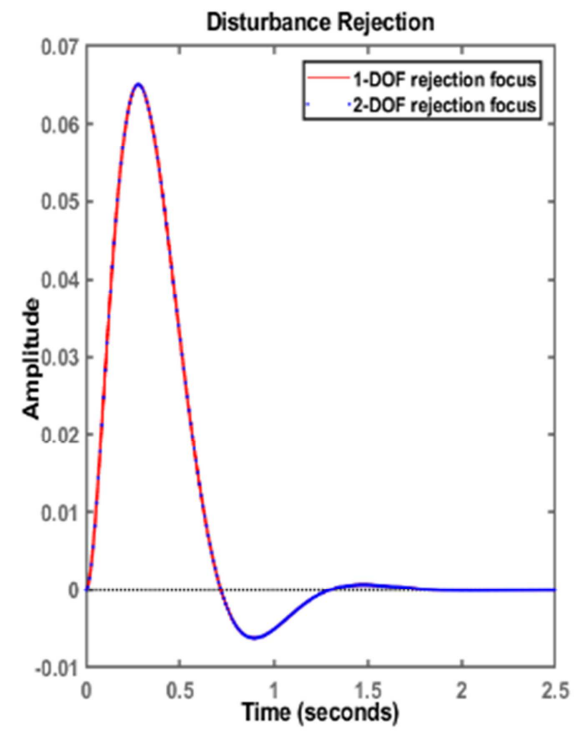

Figure 5. Comparison between $1 D O F$ and 2DOF PID controller with the same control objective.

Second, the variation effect of set point weights on the closed-loop system response under step input change is shown in Figure 6. The graphical representation reveals that gradually decreasing of $b$ causes reduction of system oscillation (Lower Overshoot), in contrast, the rising time directly proportion decreased. In addition, the disturbance

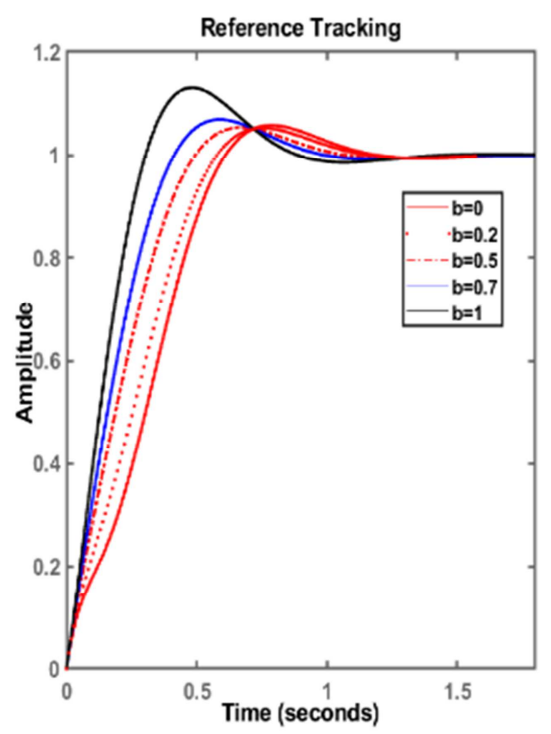

rejection response is the same at all cases. Note that, the rising time variation is considered as one of 2DOF PID filter type controller demerits. As well as, the effect on the set point tracking signal is shown in Figure 7, which cause a reshape of the error signal input to the propose controller by adding the response of $b$ to the reference tracking signal.

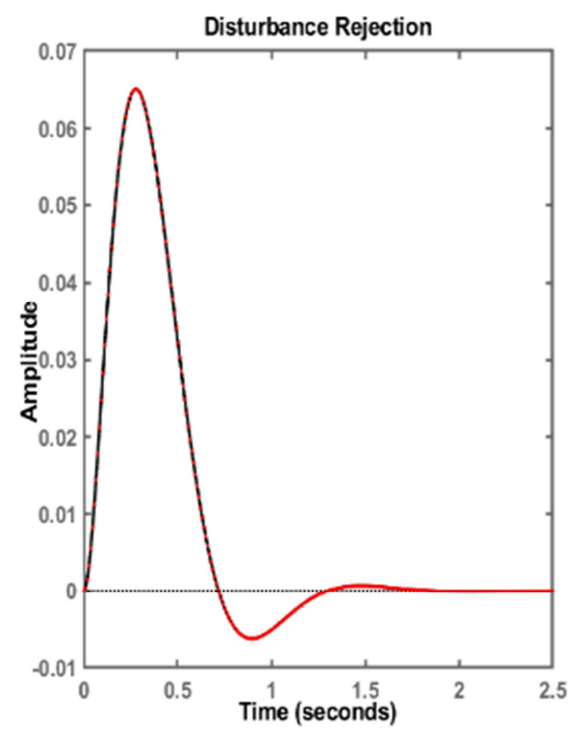

Figure 6. Effect of set point weighting variation on system response. 

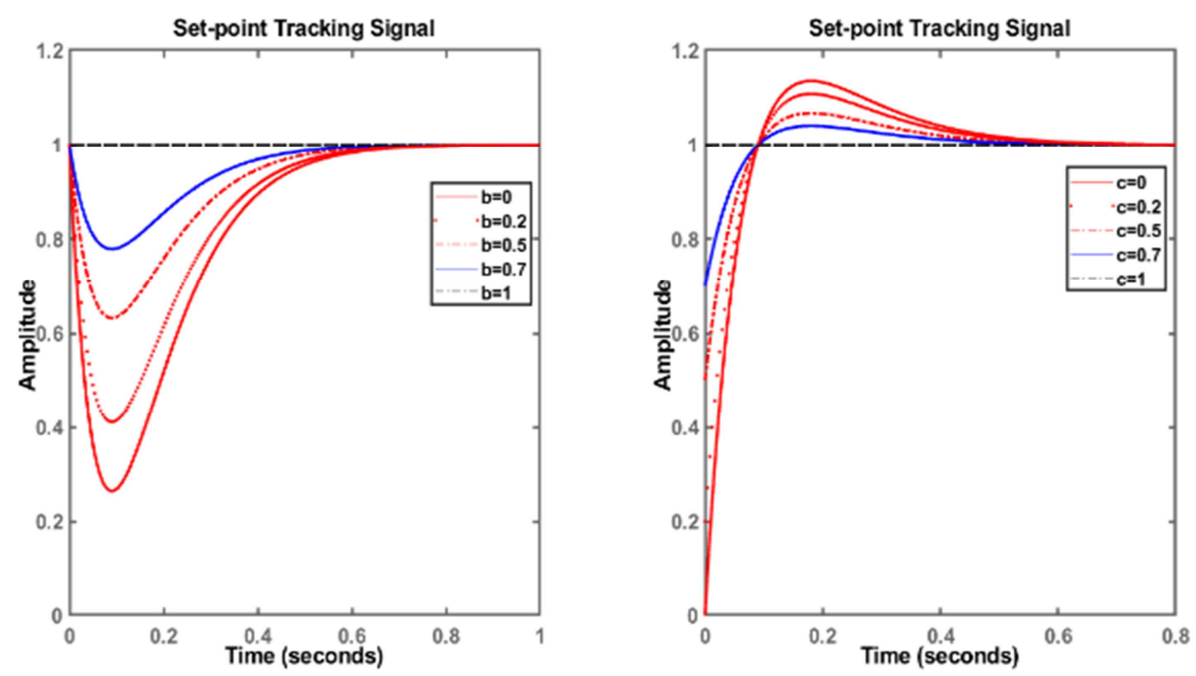

Figure 7. Set point weights variation effect on the tracking reference signal

In addition, the comparison between 1DOF PID controllers with different control objective against 2DOF PID controller is implemented at phase margin $=60^{\circ}$, thus the change of phase margin will causes a great variation at step response and load disturbance rejection as shown in Figure 8.
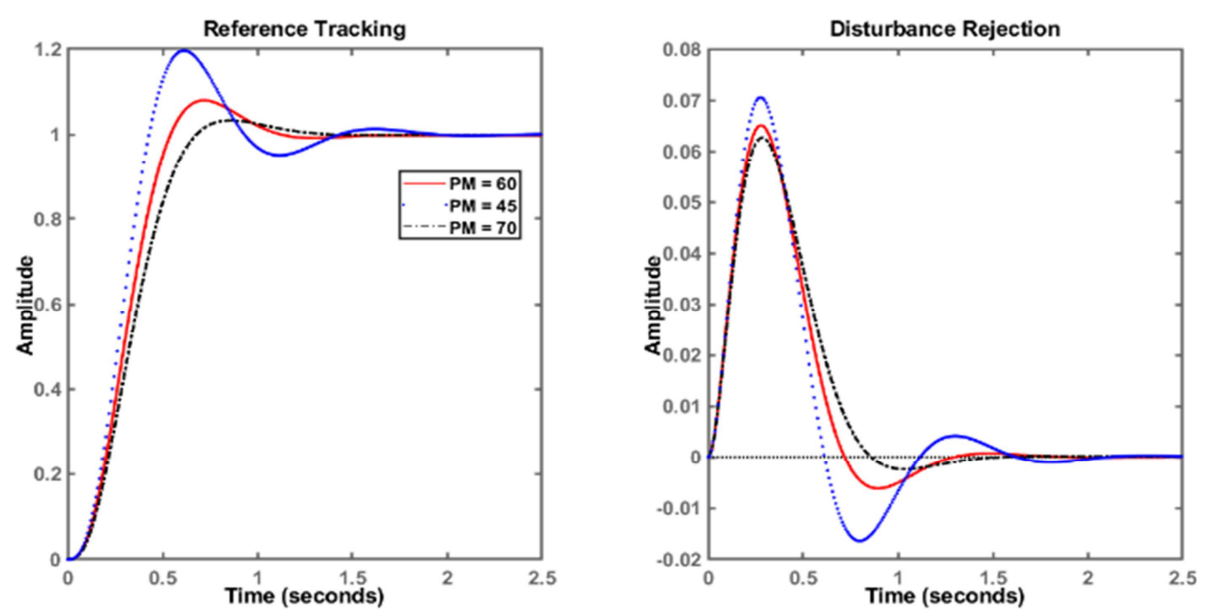

Figure 8. System response under variation of system phase margin

In other words, 2DOF PID appears as cutting-off the overshoot of the set point response. 2DOF PID controller achieved control system specification w.r.t. time response and load disturbance rejection without variation of system robustness more than 1DOF PID controller with fixed set point weighting did. On the other hand, the high set point weighting value has achieved lower rise time with higher overshoot.

The simulation results for a unit step reference and unit step load disturbance is illustrated in all the previous figures, in addition, the performance comparison between the proposed controller with other classical controller at the same control system objective is given in Table 1.

Table 1. Comparison between the effect of $1 D O F$ and 2DOF PID controller with different control objective.

\begin{tabular}{|c|c|c|c|c|c|c|}
\hline \multirow{3}{*}{$\begin{array}{l}\text { Controller } \\
\text { Type }\end{array}$} & \multirow{3}{*}{ Control Objective } & \multicolumn{5}{|c|}{ Performance Measures } \\
\hline & & \multicolumn{3}{|c|}{ Time Response } & \multicolumn{2}{|c|}{ Rejection Response } \\
\hline & & Rise Time[sec] & Settling Time[sec] & Overshoot (\%) & Peak value & Settling Time[sec] \\
\hline 1DOF & Balanced & 0.257 & 0.847 & 5 & 0.0619 & 1.24 \\
\hline $1 \mathrm{DOF}$ & Rejection Focus & 0.227 & 0.81 & 13 & 0.0651 & 1.2 \\
\hline 2DOF & Balanced & 0.767 & 0.937 & 1.2 & 0.0619 & 1.24 \\
\hline $2 \mathrm{DOF}$ & Rejection Focus & 0.877 & 1.49 & 9 & 0.0651 & 1.2 \\
\hline
\end{tabular}

\section{Controller Evaluation}

As explained in former sections, one of the alternatives to solve the tuning problem of classical PID controller was to use the optimal disturbance rejection performance and limit the rate of change in the following reference signal. In the actual control systems, the change in the set point signal is 
happened when a step change in the controlled variable is required. The effect of the set point weighting controller causes a change in the rate of set point variable to be slower.

There are several comments about using set point weighting PID controller, which are stated in the following points:

a) The proposed technique used to avoid the large overshoot is the same in both of classical 1DOF PID and 2DOF PID controller. However, both of them sharply different specifically in realization within linear and nonlinear operation conditions [31].

b) A 2DOF PID controller is capable of fast disturbance rejection without significant increase of overshoot in set point tracking.

c) 2 DOF PID controllers are also useful to mitigate the influence of changes in the reference signal on the control signal.

d) The classical control PID offers an accepted performance in only within linear framework, on the other side, 2DOF PID controller gives an accepted performance within linear and nonlinear systems.

e) The main structure of 2DOF PID controller contains the construction of classical PID within its structure. Although some researchers think, it is not a modern technique; the 2DOF PID controller enables to understand classical PID in a unique way to obtain the control system requirements.

f) The re-allocation of the zeros of the transfer function from the set point to the controlled variable is the obvious effect of the 2DOF PID control structure.

g) 2DOF PID controller is only realized effective by the feed forward compensator where the sensitivity function is small enough. As a result, in like cases, the adjustment of response through feed forward compensator is fruitless.

h) The effect of decreasing the set point weight value is indeed to make the response less oscillatory.

i) It is worth stressing that the reduction of the overshoot obviously implies that the control effort is reduced.

j) In any case, in order to avoid time-consuming experiments, tuning rules have been devised to determine explicitly a suitable value of $b$ starting from a model of the process.

$\mathrm{k}$ ) Increasing of rising time due to a lower value of $b$ is considered as a side effect of reduction of overshoot. However, there is a solution to overcome this problem by applying a variable set point weighting during the control process using any of modern control technique such as Fuzzy Logic Control or Genetic Algorithm [32, 33].

\section{Conclusion}

In this paper, a fixed set point weighting PID controller is proposed for a control structure that exploits the advantages of 2DOF PID over the classical form. The experimental study and the extensive simulation results have revealed that the performance of set point weighting PID controller offers a great chance to overcome classical PID controller limitation to obtain specific control system performance on linear and nonlinear systems as well as having the same point of a great development in digital implementation. In addition, the output of control system that has been obtained accepted overshoot with accepted disturbance rejection performance.

\section{References}

[1] Alfaro, V. M. and R. Vilanova, Control System Evaluation Metrics, in Model-Reference Robust Tuning of PID Controllers. 2016, Springer. p. 21-28.

[2] Ang, K. H., G. Chong, and Y. Li, PID control system analysis, design, and technology. IEEE transactions on control systems technology, 2005. 13(4): p. 559-576.

[3] Martelli, G., Comments on "New results on the synthesis of PID controllers". Automatic Control, IEEE Transactions on, 2005. 50(9): p. 1468-1469.

[4] Araki, M. and H. Taguchi, Two-degree-of-freedom PID controllers. International Journal of Control Automation and Systems, 2003. 1: p. 401-411.

[5] Araki, M. and H. Taguchi, Two-degree-of-freedom PID controllers. International Journal of Control, Automation, and Systems, 2003. 1(4): p. 401-411.

[6] Åström, K. J., et al., Towards intelligent PID control. Automatica, 1992. 28(1): p. 1-9.

[7] Ho, M.-T. and C.-Y. Lin, PID controller design for robust performance. Automatic Control, IEEE Transactions on, 2003. 48(8): p. 1404-1409.

[8] Åström, K. J. and T. Hägglund, The future of PID control. Control engineering practice, 2001. 9(11): p. 1163-1175.

[9] Bingi, K., et al., Fuzzy Gain Scheduled Set point Weighted PID Controller for Unstable CSTR Systems. parameters, 2017. 500: p. 6 .

[10] Mudi, R. K. and C. Dey, Performance improvement of PI controllers through dynamic set point weighting. ISA transactions, 2011. 50(2): p. 220-230.

[11] Chen, Y. and D. P. Atherton, Linear feedback control: analysis and design with MATLAB. Vol. 14. 2007: Siam.

[12] Astrom, K. and T. Hagglund, Advanced PID Control, ISAThe Instrumentation. System, and Automation Society, 2006.

[13] Dey, C., R. Mudi, and T. Lee. A PID controller with dynamic set point weighting. in Industrial Technology, 2006. ICIT 2006. IEEE International Conference on. 2006. IEEE.

[14] Visioli, A., Practical PID control. 2006: Springer Science \& Business Media.

[15] Mitra, P., C. Dey, and R. K. Mudi, DYNAMIC SET POINT WEIGHTING FOR FUZZY PID CONTROLLER. Control and Intelligent Systems, 2017. 45(3).

[16] Singh, A. K. and A. Pandey, Intelligent PI Controller for Speed Control of SEDM using MATLAB. International Journal of Engineering Science and Innovative Technology (IJESIT), 2013. 2(1). 
[17] Messner, W. C., D. M. Tilbury, and A. P. R. Hill, Control Tutorials for MATLAB ${ }^{\circledR}$ and Simulink ${ }^{\circledR}$. 1999: AddisonWesley.

[18] Salem, F. A., Controllers and Control Algorithms: Selection and Time Domain Design Techniques Applied in Mechatronics Systems Design (Review and Research) Part I. International Journal of Engineering Sciences, 2013. 2(5): p. 160-190.

[19] Tavakoli, S. and P. Fleming. Optimal tuning of PI controllers for first order plus dead time/long dead time models using dimensional analysis. in European Control Conference (ECC), 2003. 2003. IEEE.

[20] Ho, M. T. and H. S. Wang, PID controller design with guaranteed gain and phase margins. Asian Journal of Control, 2003. 5(3): p. 374-381.

[21] Mizumoto, I., H. Tanaka, and Z. Iwai. 2 DOF adaptive PID control with a parallel feedforward compensator for nonlinear systems. in Networking, Sensing and Control, 2009. ICNSC'09. International Conference on. 2009. IEEE.

[22] Alfaro, V., R. Vilanova, and O. Arrieta, Robust tuning of TwoDegree-of-Freedom (2-DoF) PI/PID based cascade control systems. Journal of process control, 2009. 19(10): p. 16581670.

[23] Leva, A. and A. Colombo. Method for optimising set point weights in ISA-PID autotuners. in Control Theory and Applications, IEE Proceedings-. 1999. IET.

[24] Esch, J., et al. Control performance index minimal tuning of set point weighted PID-Controllers for LTI plants based on convex optimisation. in Control \& Automation (MED), 2013 21st Mediterranean Conference on. 2013. IEEE.
[25] Salem, F. A. and A. A. Rashed, PID Controllers and Algorithms: Selection and Design Techniques Applied in Mechatronics Systems Design-Part II. International Journal of Engineering Sciences, 2013. 2(5): p. 191-203.

[26] Guide, G. S., Control System Toolbox ${ }^{\mathrm{TM}} .2000$.

[27] Prashanti, G. and M. Chidambaram, Set point weighted PID controllers for unstable systems. Journal of the Franklin Institute, 2000. 337(2): p. 201-215.

[28] Vilanova, R. and O. Arrieta, PID design for improved disturbance attenuation: $\min \max$ Sensitivity matching approach. International Journal of Applied Mathematics, 2007. 37(1): p. $1-6$.

[29] Tewari, A., Advanced control of aircraft, spacecraft and rockets. Vol. 37. 2011: John Wiley \& Sons.

[30] Works, M., SIMULINK: Dynamic System Simulation for MATLAB, Ver. 2. The Math Works, Inc., USA, 1997.

[31] Gamal, B. E.-d., et al., Advanced Fast Disturbance Rejection PI Controller for DC Motor Position Control.

[32] Sen, K., et al., Fuzzy Rule-Based Set Point Weighting for PID Controller, in Advances in Communication, Devices and Networking. 2018, Springer. p. 797-806.

[33] Bianchi, F. D., R. J. Mantz, and C. F. Christiansen, Multivariable PID control with set point weighting via BMI optimisation. Automatica, 2008. 44(2): p. 472-478. 